\title{
EDITORIAL Thirty years of Oncogene
}

Oncogene (2018) 37, 553-554; doi:10.1038/onc.2017.388; published online 23 October 2017

The paper by Stehelin et al. ${ }^{1}$ published in Nature in 1976 entitled 'DNA related to the transforming gene(s) of avian sarcoma viruses is present in normal avian DNA,' which demonstrates that the src sequences of the Rous sarcoma virus are derived from the host genome marks a symbolic beginning or an early major triumph and high point in molecular oncology. This work culminated in Michael Bishop and Harold Varmus being awarded the 1989 Nobel Prize in Physiology and Medicine and spawned the promise of the study of oncogenes in delineating the mechanisms of tumorigenesis. The enthusiasm generated by this and allied pioneering works from that era led to the creation of Oncogene 30 years ago.

The first papers were published in Oncogene in March 1987 under the stewardship of the founding co-Editors-in-Chief Graham Currie and John Jenkins from the Marie Curie Research Institute in Oxford and Prem Reddy from this Wistar Institute in Philadelphia. In an editorial accompanying the inaugural issue, the editors focused on the mundane details of the submission process rather than laying out a grand vision for the journal but certainly, the contemporary excitement of the field spoke for itself. The editors ended the short piece with the following anachronism, which perhaps represents how much the context of our world has changed in 30 years: 'Speed of publication is an essential characteristic of this journal and maximum use of the telephone will help minimize delays.' Notable papers in the inaugural issue included an impactful investigation into 'Exploration of ras protooncogene proteins in normal human tissues' by Furth et al., ${ }^{2}$ which reported p21ras expression most fetal and adult tissues suggesting a role in cellular proliferation and in specialized cellular functions. Kamata et al. investigated protein kinase $C$ activity in ras-resistant transformed cells and Rando et al. reported on the transactivation of the HIV virus by herpes virus. ${ }^{3,4}$

Oncogene has fulfilled its mission of being a top-notch forum for molecular oncology. The most highly cited papers from each decade of publication give an indication of how the field of molecular oncology research has evolved, grown, and the parallel contribution of this journal. A 1994 report 'Tumor-suppressor p53 is a regulator of bcl-2 and bax gene-expression in-vitro and invivo' has received nearly 2000 citations to date and remains the most cited research paper on his Oncogene's history. ${ }^{5}$ In it, Miyashita et al. show that mice deficient in p53 exhibit increases in $\mathrm{Bcl}-2$ and decreases in Bax, suggesting a molecular mechanism by which p53 regulates apoptosis in cancer. A 1999 paper entitled 'The role of cyclooxygenases in inflammation, cancer, and development' remains the most cited article from our second decade of publication. ${ }^{6}$ Williams et al. provide genetic evidence that COX-2 contributes to tumor formation and growth and suggest that both tumor and stroma-derived COX-2 may influence tumor angiogenesis and immune function. In the most recent decade of publication, a 2008 paper by Asangani et al. entitled 'MicroRNA-21 (miR-21) post-transcriptionally downregulates tumor suppressor Pdcd4 and stimulates invasion, intravasation and metastasis in colorectal cancer' is the most highly cited and describes how Pdcd4 is negatively regulated by miR-21 and demonstrates that miR-21 induces disease progression colon cancer. ${ }^{7}$
Certainly, an unstated goal 30 years ago for both the collective field molecular cancer research and Oncogene in particular was not only to enhance our relatively rudimentary understanding of the signaling pathways leading to cancer development, growth and metastases, but also to apply this knowledge to the development of new durable treatments. Considering the array of available cancer therapeutics today, the landscape of cancer treatment options 30 years ago was starkly barren. An understanding of molecular oncogenesis has resulted in impressive inroads into clinical oncology and these successes in basic discovery and translation should be celebrated by our journal and community. Pharmacological targeting of HER2 has extended survival and even provided cures to selected patients with advanced breast cancer. Imatinib, a diversified tyrosine kinase inhibitor, has had remarkable success in the treatment of CLL and GIST. Drugs that block EGFR have shown efficacy in colorectal cancer in tumors that do not harbor Kras mutations. Targeted therapy is also available for lung cancer patients with mutations in the $A L K$ and $R O S$ genes and BRAF inhibitors have been approved by the FDA for patients with BRAF-mutated melanomas. Indeed, our field has been the harbinger for personalized molecular medicine with successes that were far out of reach, if not far from the imagination, just 30 years ago. More broadly, COX-2 inhibition by aspirin and related compounds may have profound anti-cancer effects, building from the bench to the bedside.

However, with success has come an equal dose of failure. While the hope three decades ago may have been that understanding molecular oncogenesis would uncover the keys to abrogating tumor growth, the sobering reality 30 years later is that targeting oncogenes or signaling pathways in isolation will likely only lead to limited success. Indeed, targeted cancer therapies have typically been found to have efficacy in only selected patient subsets and have exhibited limited durability in most patients. Remarkably, their activity may be in part related to off-target effects. In parallel, an increased appreciation for the critical interaction of oncogenic pathways with parallel processes in the host has emerged. Indeed, even in January 1990, an editorial in Oncogene celebrating the awarding of the Nobel Prize to Bishop and Varmus, editors George Vande Woude and Prem Reddy noted that 'the amalgamation of disciplines encompassing tumor virology and biology, cytogenetics, chemical carcinogenesis and cell-growth regulation into the field of Molecular Oncology with such breathtaking speed that it is an extraordinary challenge for the research community to digest and implement'. ${ }^{8}$ The arena of oncogenesis is multidisciplinary, as must be the approach to oncogene-based therapeutics. Our contemporary understanding suggests that mutations in molecular pathways are influenced by immunity, metabolism and the microbiome and so on, and reciprocally affect these homeostatic mechanisms. A quintessential example from translational oncology of the cross-disciplinary sphere of molecular oncogenesis is the emerging data that PD-1 targeted immunotherapy has selective efficacy in gastrointestinal cancers with microsatellite instability. ${ }^{9}$ Perhaps this is already appreciated by our readers but it bears repeating that we in the cancer research community ought not to think of oncogenes in isolation from allied disciplines and as Oncogene enters its fourth decade it must expand its scope to cover these connected processes that affect tumorigenesis to stay as relevant a resource as it has been for the past 30 years. 
We take over the stewardship of this journal from Douglas Green, and will keep it at the forefront of strong cancer science for years to come.

\section{CONFLICT OF INTEREST}

The authors declare no conflict of interest.

G Miller $^{1}$ and J Stebbing ${ }^{2}$

${ }^{1}$ Departments of Surgery and Cell Biology, New York University School of Medicine, New York, NY, USA and ${ }^{2}$ Department of Surgery and Cancer, Imperial College, London, UK E-mail: George.Miller@nyumc.org

\section{REFERENCES}

1 Stehelin D, Varmus HE, Bishop JM, Vogt PK. DNA related to the transforming gene (s) of avian sarcoma viruses is present in normal avian DNA. Nature 1976; 260: 170-173.
2 Furth ME, Aldrich TH, Cordon-Cardo C. Expression of ras proto-oncogene proteins in normal human tissues. Oncogene 1987; 1: 47-58.

3 Kamata T, Sullivan NF, Wooten MW. Reduced protein kinase C activity in a ras-resistant cell line derived from Ki-MSV transformed cells. Oncogene 1987; 1: 37-46.

4 Rando RF, Pellett PE, Luciw PA, Bohan CA, Srinivasan A. Transactivation of human immunodeficiency virus by herpesviruses. Oncogene 1987; 1: 13-18.

5 Miyashita T, Krajewski S, Krajewska M, Wang HG, Lin HK, Liebermann DA et al. Tumor suppressor p53 is a regulator of bcl-2 and bax gene expression in vitro and in vivo. Oncogene 1994; 9: 1799-1805.

6 Williams CS, Mann M, DuBois RN. The role of cyclooxygenases in inflammation, cancer, and development. Oncogene 1999; 18: 7908-7916.

7 Asangani IA, Rasheed SA, Nikolova DA, Leupold JH, Colburn NH, Post S et al. MicroRNA-21 (miR-21) post-transcriptionally downregulates tumor suppressor Pdcd4 and stimulates invasion, intravasation and metastasis in colorectal cancer. Oncogene 2008; 27: 2128-2136.

8 Vande Woude Jr. GF, Reddy EP. Oncogenes and the Nobel Prize. Oncogene 1990; 5: 3.

9 Xiao Y, Freeman GJ. The microsatellite instable subset of colorectal cancer is a particularly good candidate for checkpoint blockade immunotherapy. Cancer Discov 2015; 5: 16-18. 